\title{
Innovative Configuration Design of Two-Wire Tip Mechanisms for a Tipping-Bucket Rain Gauge
}

\author{
Manuel Tumanda Tabada Jr. ${ }^{1,2, *}$, Michael Estela Loretero ${ }^{1,3}$ \\ ${ }^{1}$ Engineering Graduate Program, School of Engineering, University of San Carlos, Talamban, Cebu, Philippines \\ ${ }^{2}$ Agusan del Sur State College of Agriculture and Technology, Bunawan, Agusan del Sur, Philippines \\ ${ }^{3}$ Deparment of Mechanical Engineering, School of Engineering, University of San Carlos, Talamban, Cebu, Philippines \\ Received 23 September 2019; received in revised form 17 November 2019; accepted 18 January 2020 \\ DOI: https://doi.org/10.46604/ijeti.2020.4772
}

\begin{abstract}
This paper evaluates the measurement accuracy of the three designs of an innovative Tipping-Bucket Rain Gauge (TBRG) tip mechanism. A water-level detecting circuit is used to replace the conventional reed switch sensor, which addresses the disadvantages of the magnetic sensing method that became a factor in quantification uncertainties. The TBRG configuration designs were the bucket-feed, which detects the presence of water inside the bucket, and the bottom-feed and the center-feed, which use the tip-impact method in measuring rainfall. The constant flow method is used in the experimentation. The bucket-feed shows potential in precision rainfall measurement for having $-3.84 \%$ and $-2.68 \%$ accuracy errors at $6 \mathrm{~mL} / \mathrm{min}$ and $11 \mathrm{~mL} / \mathrm{min}$ respectively, without correction algorithm applied. The tip-impact application for the bottom-feed and the center-feed resulted in a higher error percentage from the volumetric flow samples. The result indicates that actual detection in the bucket brings more measurement accuracy than the tip-counting technique.
\end{abstract}

Keywords: rain data acquisition, tipping-bucket rain gauge, tip mechanism, water level detection

\section{Introduction}

Rainfall measurements have been easily made attainable through rain gauges. Catching and non-catching types of this instrument were crafted utilizing different measuring techniques. Known applications were from the Tipping-Bucket Rain Gauge (TBRG), weighing gauges and impact disdrometers. These gauges were tested and proven its efficacy through an inter-comparison measurement assessment conducted by the World Meteorological Organization (WMO) in 2007.

The Tipping-Bucket Rain Gauge (TBRG), due to its measurement accuracy potential has become one of the forefronts in rainfall metrology; twelve different global designs of this type of rain gauge were included in the assessment by the WMO [1]. The main components of this instrument were the pivotal buckets and the funnel. For its general operation, the rainwater travels onto the funnel, filling out each of the sub-compartment in the bucket alternately. As the continuous rainwater drops into the compartments, a sideward alternate movement which known as the "bucket tip" has been created. The tip alternation of the bucket is monitored by an automated tip-counting system. The bucket tip count became the measurement basis of this type of instrument.

The magnetic sensing system was used for the tip counting, as what can be found on most references in the TBRG literature. Magnetic sensors utilized were proximity sensors [2], Hall-effect sensors [3], and reed switch sensors applied on

\footnotetext{
* Corresponding author. E-mail address: doitabada@gmail.com
} 
either single [4-7] or dual installation [8-10] in the rain gauge instrument itself. As stated, most TBRG uses reed switch as the magnetic sensor, making it the conventional tip counting component for this type of instrument. However, recorded drawbacks of this sensing method include magnetic interference when exposed near to any ferromagnetic materials and electromagnetic pulses [11], resulting in an unwanted tip detection from the instrument. A scientific fact regarding the effect of magnetism through temperature variations is also considered. Reed switches mainly have contact bounce issues [12-13].

Other tip-counting methods for the TBRG were presented by $[2,11]$ using a proximity sensor and the Hall Effect sensor by [3, 14], but still covered by the electromagnetic interference problem. The potentiometer type TBRG presented by [15] also has rotary problems when exposed to dust or rust. A two-wire electronic sensing technique for the TBRG was introduced [16] to address most of the stated problems. This method technically performs tip detection similar to that reed switch sensing process but using electronic contact sensing instead of magnetic components. However, the system's measuring capability was not maximized as it was only presented on a single configuration. Also, it was not tested on lower rainfall intensity estimations, which has higher produced rainfall intensity errors [6]. Since the primary process for this scheme is the physical contact between wire tip probes, improper design could lead to tip detection inaccuracy. This paper aims to design and implement a TBRG using water level detection that will optimize the reading accuracy of the TBRG through electronic tip mechanism configurations.

\subsection{The contact-type water level detector}

Liquid level sensors were commonly utilized on storage tanks and water reservoirs to monitor water depth. Upgrades on this technology for the past years resulted in contact or submersible and non-contact type sensor classifications. Floating sensors [17-18], pressure-based sensors [19-21], ultrasonic level sensors [22] and capacitive sensors [23-28] were among the known technology of these types. Another common water level sensor is the switch-type sensor using the concept of electrical conductivity of water. The one presented by [16] uses two contacts as impedance measurement probes. If the fluid level touches the two probes, low impedance will be attained by the sensor. The low-cost and simple circuitry of this type of sensor has been known to be robust and can be fitted on any compact designs. Due to its contact sensing nature, it has high water detection accuracy compared to other sensors. With the sensing reliability of the circuit, it was found out to be applicable in other water sensing applications and not limited to water level detection. In this case, the concept was used as the tip quantification method for TBRG.

The utilized circuit is composed of low-cost electronic components shown in Fig. 1. A single circuitry is comprised of a Darlington Pair configuration of two PNP Transistors (T1 and T2), resistors (R1, R2, and R3), and a set of tip and ground (common) conducting probes as input terminals. When these conducting probes make contact with water, it allows electrical current. The variable resistor R3 serves as the probes sensitivity adjuster.

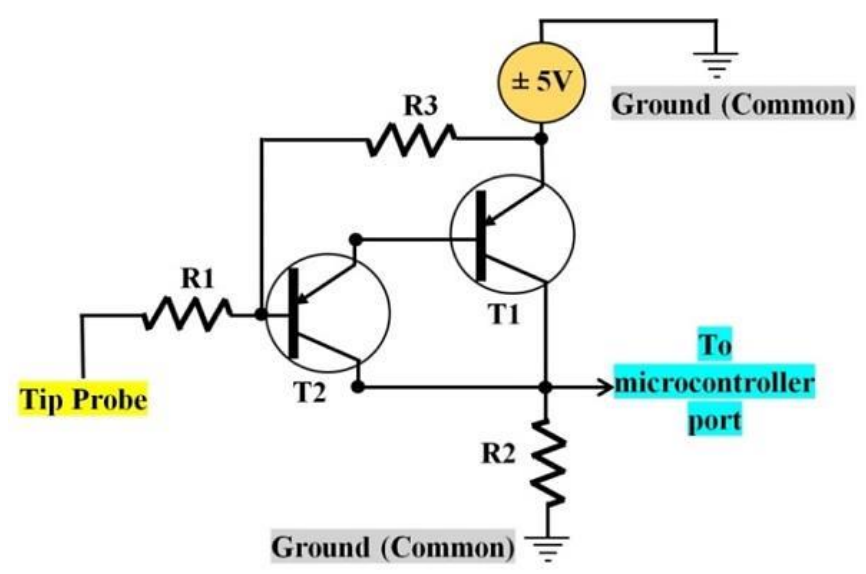

Fig. 1 The water-detecting circuit 


\section{Materials and Methods}

\subsection{The tip sensing designs}

The general aim in the process of the proposed tip counting scheme is to allow a temporal contact between the two probes of the water level sensing circuit on every bucket alternations whether physically or through water conductivity. This contact will create conduction and produce a DC pulse going into the circuitry. It was highly expected that the water inside the bucket will initiate contact directly or indirectly. With this target concept, three tip mechanism configuration designs were developed.

For the overall setup, two water-sensing circuits are utilized for the purpose of monitoring the state of the two sub-compartments (left and right) of the bucket. The two circuits have their own set of probes (ground and tip) that were installed in each compartment.

The bottom-feed design shown in Fig. 2 has sensing probes stationed underneath the bucket, with $1 \mathrm{~mm}$ separation between the common and tip probe. The probes used in this design are a solid $0.5 \mathrm{~mm}$ conducting wire with soldered flat metal tip. The tip probes (green and yellow), are expected to make contact with the common probe (black) every time the bucket makes a downward alternation movement due to the weight of the water. The contact sensitivity was the main reason for the probe separation.

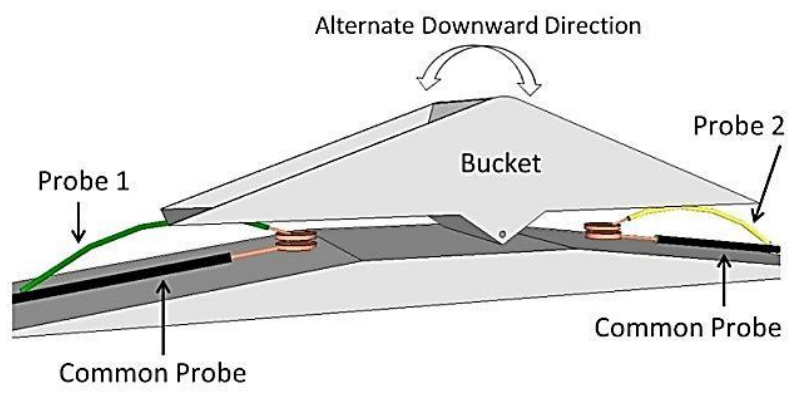

Fig. 2 TBRG bottom-feed configuration

The center-feed design makes use of a thin conducting plate instead of using the copper conductor wire. A hook-type probe shown in Fig. 3 is attached to the center of the bucket in order to be in accordance with the direction of the bucket. In every alternation, the sideways movement of the hook makes contact with the stationary thin plates installed underneath the circuit case. These plates are attached to the sensing circuit. The upper thin plates represent the tip probes while the lower plates are the common probes. Every left and right pan movement of the bucket touches the still plates.

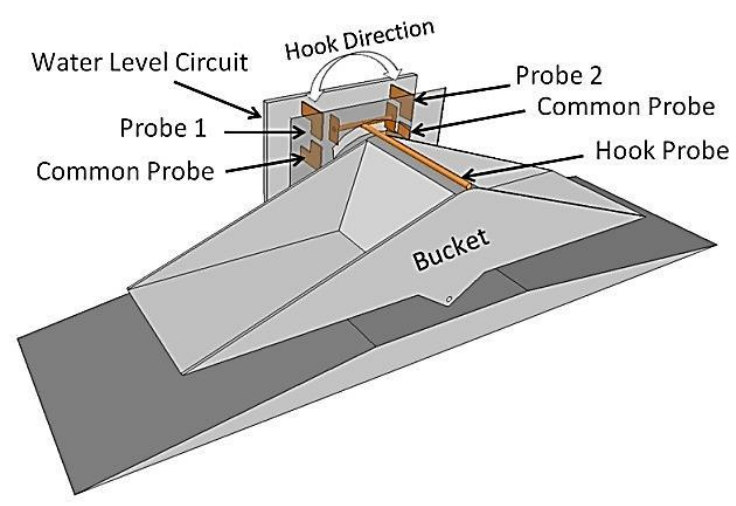

Fig. 3 TBRG center-feed configuration

The bucket-feed design does not primarily rely on the movement of the bucket; instead, it detects the actual water inside the bucket. The stationary probe sensors shown in Fig. 4 hang up tightly in a diagonal manner pointed down at the bucket. These probes are preferred to be flexible $0.5 \mathrm{~mm}$, solid conductors, for easy adjustments to where the tip should be pointed. 
The tips of the probes are approximately positioned to the end part where the maximum amount of water can possibly be captured just before the bucket tips. The "bucket-tipping" amount of water is estimated as the threshold volume.

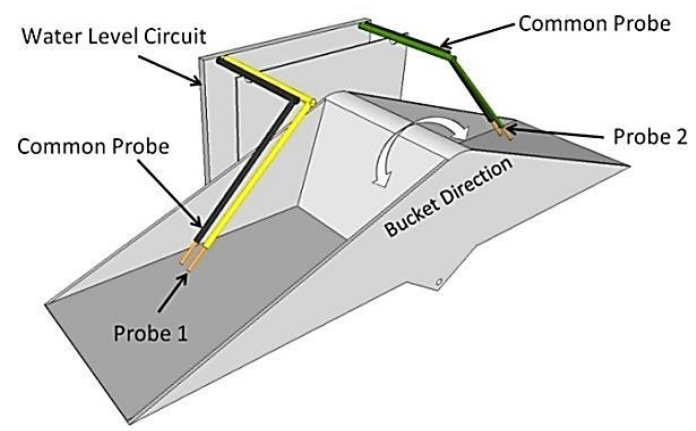

Fig. 4 TBRG bucket-feed configuration

\subsection{Pulse conversion}

For the voltage readings, the Analog-to-Digital Converter (ADC) reading value of the microcontroller module is considered. Because of the 8-bit analog input of the ADC, a 0 to 1023 reading is generated with the higher value corresponding to the 5 volts DC. By using the ratiometric conversion, the ADC output can then be converted into its corresponding DC voltage (VDC) value using:

$$
V D C=\frac{(A D C * 5)}{1023}
$$

\subsection{Logical operation}

The diagram in Fig. 5 shows the intended application of water sensing on the TBRG. As the bucket tips downward and upward alternately, it creates the contact between the tip and ground probes positioned in the bucket. Two of the configurations presented use the actual contact of the probes for bucket tip detection, and the other using the electrical conductivity of water. The created contact between the probes is the basis for the logical operation. When the probes were in an "off-hook" state, no DC pulse will be detected by the microcontroller and logic " 0 " will be recorded; otherwise, the microcontroller reads it as logic "1".

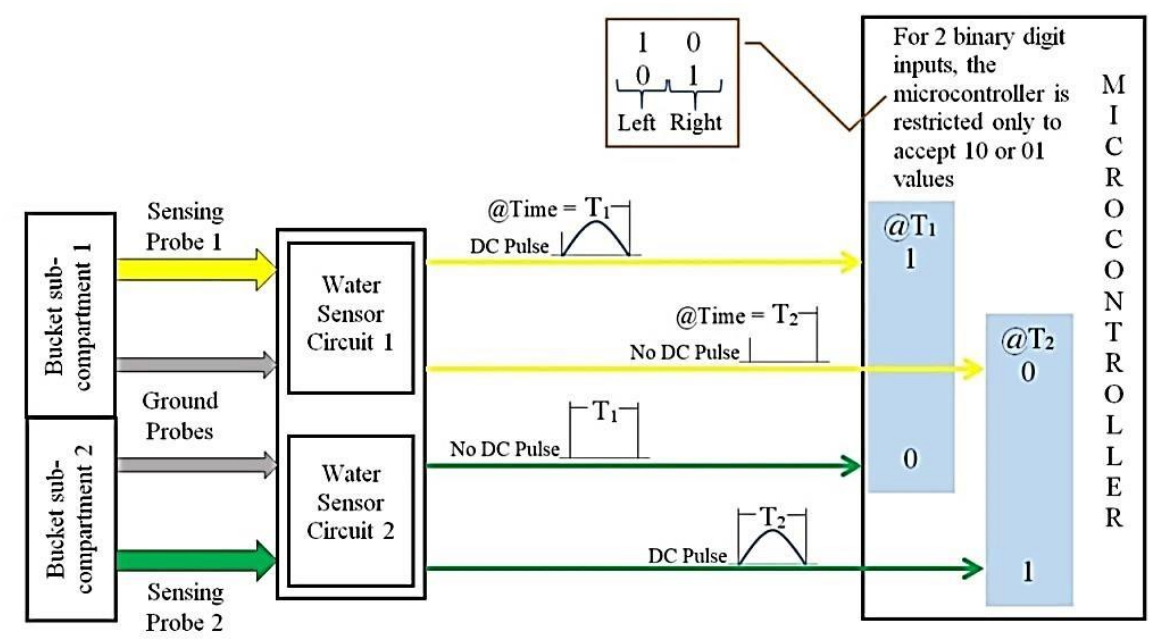

Fig. 5 The applied logical concept

Every alternation results in binary digit inputs of either a 10 or 01 status, meaning the other compartment tips-on, the other compartment is idle. This is the ideal sequence needed by the microcontroller program to pinpoint the specific probe that made contact with the ground probe, which also identifies the bucket tips made, either through the downward impact movement or 
due to soaking of both ground probes and the sensor probes. By applying the "if and only if' restriction in the program, the microcontroller only identifies tip detection if the 01 and 10 conditions will meet from the output pulses produced by the two water sensors. With this restriction, binary values other than the stated conditions were considered void and not counted as a bucket tip.

The program also includes a buffer that prevents detection from the same bucket sub-compartment accidentally tipping twice to avoid tip counting overestimation. This is because the microcontroller was limited to detect an alternate sequence, hence, the same sequence reading within two consecutive timestamps was assumed to have the same bucket status. As for the contact sensitivity of the probes, the resistor R3 of the water sensing circuit (Fig 1) can be adjusted to a hundred kilo Ohms (k $\Omega$ ) value.

\section{Experimental Setup}

This study is limited to the tip detection accuracy of the instrument and does not include the absolute measurement precision of the system on the actual rainfall. Only the performance comparison based on the constant rate of water flow samples were applied. Constant flow method was the recommended used scheme in rain gauge calibration [29] conducted by related researches and the WMO as well. In this experiment, the resulting flow rate from the pumps was carefully tested and verified in order to establish the rate values. The values, however, do not follow any standard flow rate samples because there were no established standard rates due to the explicit variability of rainfall in actual conditions. In fact, some conducted rainfall calibrations were done using variable water pumps applying flow rates lower than $18 \mathrm{ml} / \mathrm{min}$ [30] or a range of 24-100 $\mathrm{ml} / \mathrm{min}$ [31] or as high as $200 \mathrm{~mm}^{-\mathrm{h}}$ [1]. Others would use burettes as one of its rain gauge calibration method [32]. But due to the unavailability of calibrated variable water pumps during the conduct of the experiment, configured fixed-low rate water pumps were used to produce slow-paced droplets.

The whole experimentation process (Fig. 6) was to turn-on the water pump and produce water droplets, fill the bucket compartment, detect which bucket contains water before it alternately tips-off, produce a DC pulse, convert each pulse into rainfall data and lastly, record the activity. The process was repeated initiated on every bucket alternation in the entire event. Two sets of experiment process were done, the minute event and the set of trials. The minute event, in which 15 of these were conducted in each trial set, records the number of tips the bucket made and the total volume in 1-minute. The trial sets records the total mean volume in each set of events. 16 trials were conducted for the three TBRG designs.

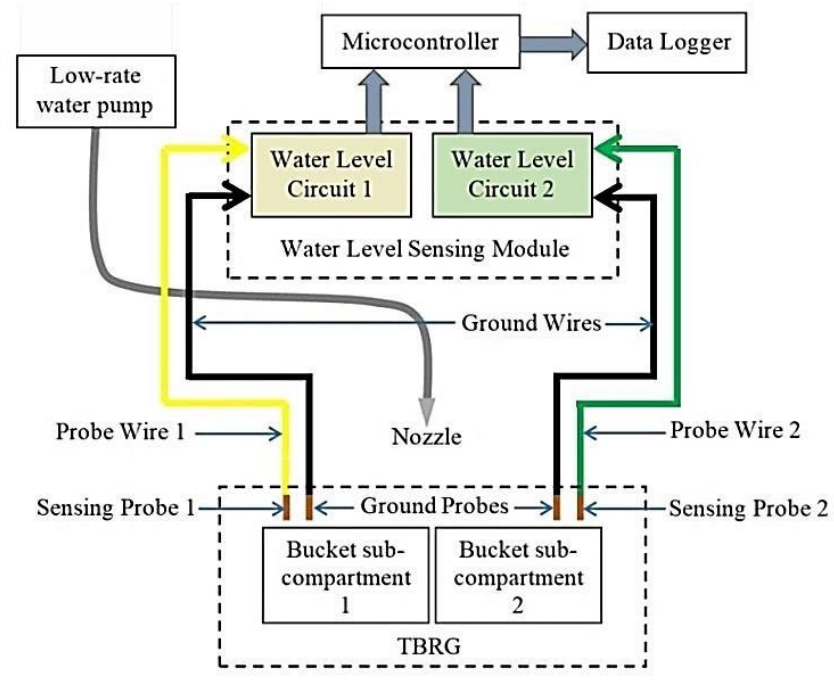

Fig. 6 Block diagram of the experimental setup

Flow rates of $2.5 \mathrm{~mL} / \mathrm{min}, 6 \mathrm{~mL} / \mathrm{min}$ and $11 \mathrm{~mL} / \mathrm{min}$ were applied for probe detection sensitivity testing. These values were not specifically chosen, but rather utilized for the reason that these were the produced flow from the available water 
pumps, verified by measuring the flow under a 1-minute interval at the hose nozzle. In addition, the nature of the study is to verify the volumetric $(\mathrm{ml})$ measurement and not the rainfall intensity ( $\mathrm{RI}$ in $\mathrm{mm}^{-\mathrm{h}}$ ), thus, it does not necessarily apply the importance of the wider variable range of flow samples. The probe detection is the most important aspect of the experiment, which was analogous to the magnetic detection sensitivity for the commercial ones. Low or too much sensitivity of the probes could lead to higher inaccuracy measurement for the reason that the entire computations for the measurements rely on the detection status of the bucket. The measurement result from the quantified water is then compared to the sample volume. The approximated volume of the TBRG is computed using the basic tip count-to-volume relation:

$$
V_{T}=\sum_{i=1}^{n} V_{i}
$$

where $\boldsymbol{V}_{\boldsymbol{T}}$ is the accumulated volume from the tip volume $V(1.2 \mathrm{~mL})$ based on the total number of tips $n$, and rain depth $\boldsymbol{R}_{\boldsymbol{d}}$ can be expressed by:

$$
R_{d}=\frac{V_{T}}{A}
$$

where $A$ is the cross-sectional area of the orifice (122 mm funnel orifice). These values lead to the approximation of rain intensity $R I_{A}$ in minute $t$, i.e.,

$$
R I_{A}=R_{d} t^{-1}
$$

As each quantification contained a fix value of input flow rate samples and average values of the resulting measured volume of water, the measurement performance can then be approximated through percentage relative error $\left(e_{r e l}\right)$ shown in Eq. (5) using the measured $\mathrm{RI}\left(R I_{m}\right)$ and the reference $\mathrm{RI}\left(R I_{r e f}\right)[33]$ based from the identified flow rates.

$$
e_{r e l}=\frac{R I_{\mathrm{m}}-R I_{r e f}}{R I_{r e f}} \times 100
$$

\section{Results and Discussion}

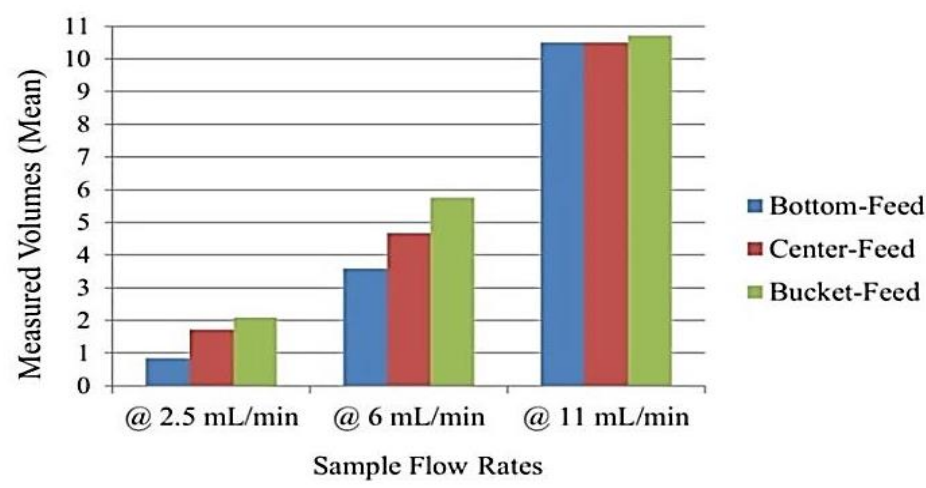

Fig. 7 The average measured volume for each TBRG design

The efficacy of the designed TBRG mainly relies on the contact sensitivity of the probes. The test results shows the relevance of contact sensitivity on this study and the bottom-feed configuration was most affected to it. The spacing of $1 \mathrm{~mm}$ between the probes underneath the bucket becomes the threshold distance. The electrical conductivity of water was a factor in the tip detection sensitivity below the threshold spacing. Similarly, the sensing error of the center-feed was also due to the inability of the probes to make full contact. With the lever-type contact, this configuration needs design precision in the arrangements of the contacts, which was not fully attained in this study. The width and orientation of the thin plates offer difficulty in making a compact circuitry, unlike its reed switching counterpart which has a lightweight and smaller circuit 
board which should be one of the main features of a rain gauge. The bottom and center-feed designs rely on the magnitude of the downward impact made by the water-filled bucket. The 2.5 and $6 \mathrm{~mL} / \mathrm{min}$ sample flow rates (Table 1) produce only small drops on a longer interval within the 1-minute timestamp. This triggers the downward movement of the bucket at a lesser force that resulted in several lapses in detecting the tips made, thus resulting in higher relative errors of $-66 \%$ and $-39.95 \%$ for the bottom-feed, and $-30.51 \%$ and -21.93 for the center-feed. Only with the $11 \mathrm{~mL} / \mathrm{min}$ sample flow rate where the tip-impact designs have similar outcomes (Fig. 7) and resulted in a low error percentage of $-4.36 \%$ tip reading accuracy.

Table 1 Reading accuracy

\begin{tabular}{|c|c|c|c|c|}
\hline Design & & $@ 2.5 \mathrm{~mL} / \mathrm{min}$ & $@ 6 \mathrm{~mL} / \mathrm{min}$ & $@ 11 \mathrm{~mL} / \mathrm{min}$ \\
\hline \multirow{4}{*}{ Bottom-feed } & Mean Volume* & 0.84 & 3.6 & 10.52 \\
\cline { 2 - 5 } & Calculated $R I$ Mean** & 0.15 & 0.65 & 1.91 \\
\cline { 2 - 5 } & Reference $R I^{* *}$ & 0.45 & 1.09 & 2.00 \\
\cline { 2 - 5 } Center-feed & Relative Error\% $\left(e_{\text {rel }}\right)$ & -66.06 & -39.95 & -4.36 \\
\cline { 2 - 5 } & Mean Volume* & 1.72 & 4.68 & 10.52 \\
\cline { 2 - 5 } & Calculated $R I$ Mean** & 0.31 & 0.85 & 1.91 \\
\cline { 2 - 5 } & Reference $R I^{* *}$ & 0.45 & 1.09 & 2.00 \\
\cline { 2 - 5 } Bucket-feed & Relative Error\% $\left(e_{\text {rel }}\right)$ & -30.51 & -21.93 & -4.36 \\
\cline { 2 - 5 } & Mean Volume* & 2.09 & 5.77 & 10.71 \\
\cline { 2 - 5 } & Calculated $R I M e a n * *$ & 0.38 & 1.05 & 1.95 \\
\cline { 2 - 5 } & Reference $R I^{* *}$ & 0.45 & 1.09 & 2.00 \\
\cline { 2 - 5 } & Relative Error\% $\left(e_{\text {rel }}\right)$ & -15.76 & -3.84 & -2.68 \\
\hline
\end{tabular}

On outdoor conditions, corrosion can affect the tip of the probes so a much closer pressing between the probes is required in order to make a contact. The contact sensitivity can be increased on either of the two steps: 1) increasing the value of R3 in the minimum value of 50 kilo ohms $(\mathrm{k} \Omega)$ for water conduction sensitivity, and 2) decreasing the distance separation of the probes. The water conductivity and micro-vibrations between the probes produce multiple contact detection that creates an erroneous overestimation on the tip count if the distance had gone below the threshold spacing. Software correction was done to solve the problem of repeated tip contacts due to too much sensitivity or vibrations of the probes. But this doesn't resolve the downward impact issues of less water flow intensity.

The bucket-feed, on the other hand, has a stable detection and consistent on the tip reading. The reason behind the precision of this configuration is that it does not rely on the tip impact of the probes but rather detects the presence of water inside the probe. Because of the top positioning of the probes, it detects the captured water in the bucket just before it spills out of the instrument. And since each tip has a measurable amount of water volume (1.2 $\mathrm{mL}$ for the utilized instrument), applying the appropriate error correction in the software could produce much better measurement accuracy. The lowest sample volume $(2.5 \mathrm{~mL} / \mathrm{min}$ ) resulted to $-15.76 \%$ relative reading error as it was physically observed that on larger drop intervals (at $1.2 \mathrm{~mL}$ tip resolution), the water does not directly slip down at the bottom but rather stays in the center-slant area at the water receiving part of the bucket. The next drop pushes the stagnated water flows to the bottom, accumulating the water in the bucket until it slowly reached the sensing probe. With this case, the density of the solid probe conductor and water does not make it instantly blend together, causing the circuitry to miss the water detection until it tips-off alternately as the sensor probe was placed at the end part of the bucket. This situation can be compared to the faster filling of the bucket through higher volumetric flow pouring on as it resulted in only $-3.84 \%$ and $-2.68 \%$ reading accuracy error.

\section{Conclusions}

The result of this study shows an effective design for a TBRG using the water level detecting circuit. The application has a similar method from the conventional reed switching technique used on commercial TBRG. The main difference between the two methods is that the presented design is not affected by any magnetic interference, outdoor temperature, and other electronic contact switch drawbacks. 
Sensitivity and design effectivity are the main factors in the optimization of the volumetric measurements as they causes to higher accuracy error of $-66.06 \%,-30.51 \%$ and $-15.76 \%$ from the bottom-feed, center-feed and bucket-feed respectively at $2.5 \mathrm{~mL} / \mathrm{min}$ rate, which are the lowest among the volumetric flow samples. Like other TBRG designs, the measurement of the presented prototypes gets more accurate with the increase of the sample flow rates. However, with only 3 volumetric flow samples, it is not clearly seen on what level of accuracy that the presented instrument can measure. The bucket-feed design stands as a potential prospect in precision measurement for rainfall as it has only $-3.84 \%$ accuracy error for the $6 \mathrm{~mL} / \mathrm{min}$ rate and only $-2.68 \%$ at $11 \mathrm{~mL} / \mathrm{min}$ sample, given that no error correction algorithm applied in the experiment.

Apart from determining the appropriate configuration for the tip detection of a two-wire TBRG design, an important result of this study also clarifies the concept going into the instrument's measurement accuracy. As the experimental findings highlights the effectiveness of the bucket-feed design, it clearly shows that TBRG measurements should not mainly focus on how many tips detected but rather emphasizing the water content of each bucket. The main reason why electromagnetic interference and temperature have negative effects on common TBRG tip mechanism is that the technique only relies on magnetism for bucket tip counting and neglected the rainwater drenched into the bucket.

With these, the following points are summarized:

(1) The water level sensor as tip counter for the TBRG instrument is found to be effective

(2) The tip impact method applied by the bottom-feed and center-feed are effective only on higher flow rate intensities because of its reliance to the downward movement of the bucket.

(3) The direct water detection of the center-feed design is the main reason for its lesser volume measurement error.

(4) The bucket alternation may be useful in catching and ditching the water out of the container, but the measurement should rely on the water inside the bucket.

\section{Conflicts of Interest}

The authors declare no conflict of interest.

\section{References}

[1] L. G. Lanza and E.Vuerich, “The WMO field Intercomparison of rain intensity gauges,” Atmospheric Research, vol. 94, no. 4, pp. 534-543, December 2009.

[2] F. L. Conti, D. Pumo, A. Incontrera, A. Francipane, G. L. Loggiaand, and L. V. Noto, “A weather monitoring system for the study of precipitation fields, weather, and climate in an urban area," In 11th International Conference on Hydroinformatics, pp. 1-8, 2014.

[3] M. G. Syahrul, "Design and implementation of tipping-bucket rain gauge," In 1st International Conference on Informatics and Computational Sciences, pp. 195-200, 2017.

[4] B. A. Indunil and H. A. P. K. Hettiarachchi, "Automated rain gauge station with a GSM data transmission link," In 2nd International Conference on Industrial and Information Systems, pp. 387-392, 2007.

[5] X. C. Liu, T. C. Gao, and L. Liu, "A comparison of rainfall measurements from multiple instruments," Atmospheric Measurement Technique, vol. 6, pp. 1585-1595, 2013.

[6] P. Muñoz, R. Célleri, and J. Feyen, "Effect of the resolution of tipping-bucket rain gauge calculation method on rainfall intensities in an andean mountain gradient," Water, vol. 8, pp. 534, 2016.

[7] T. K. V. Raghava and S. P. Wani, "Internet enabled tipping bucket rain gauge," In 2014 International Conference on Computer Communication and Informatics, 2014.

[8] V. S. Shedekar, K. W. King, N. R. Fausey, A. B. O. Soboyejo, R. D. Harmel, and L. C. Brown, "Assessment of measurement errors and dynamic calibration methods for three different tipping-bucket rain gauges," Atmospheric Research, vol. 178-179, pp. 445-458, 2016.

[9] M. Stagnaro, M. Colli, L. G. Lanza, and P. K. Chan, "Performance of post-processing algorithms for rainfall intensity using measurements from tipping-bucket rain gauges,” Atmospheric Measurement Technique, vol. 9, pp. 5699-5706, 2016. 
[10] A. Tokay, P. G. Bashor, and V. L. McDowell, "Comparison of rain gauge measurements in the Mid-Atlantic Region," American Meteorological Society, pp. 553-564, 2010.

[11] R. K. Das and N. R. Prakash, "Design of an improvised tipping bucket rain gauge for measurement of rain and snow precipitation," International Journal of Instrumentation Technology, vol. 1, no. 1, pp. 44-59, 2011.

[12] P. Regtien and E. Dertien, "Inductive and Magnetic sensors," Sensors for Mechatronics 2nd Edition, pp. 145-182, 2018.

[13] L. X. Zhang, Y. Itoh, M. Yoshizawa, and K. Suzuki, "Basic phenomena of contact bounce in the electrical switching system," Elsevier Studies in Applied Electromagnetics in Materials, vol. 6, pp. 609-612, 1995.

[14] J. C. B. Lopez and H. M. Villaruz, "Low-cost weather monitoring with online logging and data visualization," In 8th IEEE International Conference Humanoid, Nanotechnology, Information Technology, Communication and Control, Environment and Management, 2015.

[15] M. M. Rashid, M. Rabani, M. Romlay, and M. M. Ferdaus, “Development of electronic rain gauge system," International Journal of Electronics and Electrical Engineering, vol. 3, no. 4, pp. 245-249, 2015.

[16] M. T. Tabada and M. E. Loretero, "Application of a low-cost water level circuit for an accurate pulse detection of a tipping bucket rain gauge as an alternative method for reed switch sensors," Environmental Monitoring and Assessment, vol. 191, no. 5, 2019 .

[17] A. Broring, P. Beltrami, R. Lemmens, and S. Jirka, "Automated integration of Geosensors with the sensor web to facilitate flood management," Approaches to Managing Disasters-Assessing Hazards, Emergencies and Disaster Impacts, pp. 65-86, 2012.

[18] B. Kumar and N. Mandal, "Study of an electro-optic technique of level transmitter using Mach-Zehnder Interferometer and float as primary sensing elements," Proceeding of IEEE Sensors Journal, vol. 16, no. 11, pp. 4211-4218, 2016.

[19] R. Greswell, P. Ellis, M. Cuthbert, R. White, and V. Durand, "The design and application of an inexpensive pressure monitoring system for shallow water level measurement, tensiometry, and piezometry,” Journal of Hydrology, vol. 373, pp. 416-425, 2009.

[20] Z. Liu and C. W. Higgins, "Does temperature affect the accuracy of vented pressure transducer in fine-scale water level measurement?," Geoscientific Instrumentation, Methods and Data System, vol. 4, pp. 65-73, 2015.

[21] J. Vetelino and A. Reghu, "Introduction to Sensors," Florida: Taylor and Francis Group, 2011.

[22] B. Su and X. Ma, "Water Level Sensor Based on a New design structure for irrigation water measurement," IFAC Proceedings Volumes, vol. 43, no. 26, pp. 39-44, 2010.

[23] A. Qurthobi, R. F. Iskandar, A. Krisnatal, and Weldzikarvina, "Design of capacitive sensor for water level measurement," Journal of Physics: Conference Series, vol. 776, 2016

[24] F. Reverter, L. Xiujun, and G. C. M. Meijer, "Liquid level measurement system based on a remote grounded capacitive sensor," Sensors and Actuators A: Physical, vol. 138, no. 1, pp. 1-30, 2007.

[25] J. Wei, C. Yue, Z. L. Chen, Z. W. Liu, K. A. A. Makinwa, and P. M. Sarro, "Implementation and characterization of a femto-farad capacitive sensor for pico-liter liquid monitoring," Proceedings of the Eurosensors XXIII Conference, pp. 120-123, 2009.

[26] J. Baoquan, Z. Zeyu, and Z. Hongjuan, "Structure design and performance analysis of a coaxial cylindrical capacitive sensor for liquid-level measurement," Sensors and Actuators A: Physical, vol. 223, pp. 84-90, 2015.

[27] K. Chetpattananondh, T. Tapoanoi, P. Phukpattaranont, and N. Jindapetch, "A self-calibration water level measurement using an interdigital capacitive sensor," Sensors and Actuators A: Physical, vol. 209, pp. 175-182, 2014.

[28] K. Loizou, and E. Koutroulis, "Water level sensing: State-of-the-art review and performance evaluation of a low-cost measurement system,” Measurement, vol. 89, pp. 204-214, 2016.

[29] L. G. Lanza, M. Leroy, C. Alexadropoulos, L. Stagi, and W. Wauben, "WMO Laboratory Intercomparison of Rainfall Intensity Gauges-Final Report,” Instrumental Department, INSA-IO, KNMI: De Bilt, Netherlands, 2005.

[30] M. Colli, L. G. Lanza, P. La BArbera and P. W. Chan, "Measurement accuracy of weighing and tipping-bucket rainfall intensity gauges under dynamic laboratory testing," Atmospheric Research, vol. 144, pp 186-194, 2014.

[31] S. Wijonarko, Sensus \& Maftukhah, Tatik, "Instrumentation system for water balance measurements on Serkuk Subbasin, Kubu Watershed, Belitung,” Proceedings of AIP Conference, vol. 1746, no. 1, 2014.

[32] R. A. Hodgkinson, T. J. Pepper, and W. D. Wilson, "Evaluation of Tipping Bucket Rain Gauge Performance and Data Quality," Bristol, Environment Agency, 2004.

[33] UNI 11452: 2012, "Hydrometry-Measurement of rainfall intensity (liquid precipitation)-Metrological requirements and test methods for catching type gauges,” Ente Nazionale Italiano di Unificazione, Milano, Italy, 2012.

Copyright $(\mathrm{C}$ by the authors. Licensee TAETI, Taiwan. This article is an open access article distributed under the terms and conditions of the Creative Commons Attribution (CC BY-NC) license (https://creativecommons.org/licenses/by-nc/4.0/). 